\title{
Neutrino Emission from Supermassive Binary Black Hole Mergers
}

\section{Ilja Jaroschewski, ${ }^{a, b, *}$ Oliver de Bruijn, ${ }^{a, b}$ Julia Becker Tjus, ${ }^{a, b}$ Peter L. Biermann, ${ }^{c, d}$ Imre Bartos ${ }^{e}$ and Wolfgang Rhode ${ }^{f}$}

${ }^{a}$ Ruhr-Universität Bochum, Theoretische Physik IV: Plasma-Astroteilchenphysik, Universitätsstrasse 150, 44801 Bochum, Germany

${ }^{b}$ Ruhr Astroparticle and Plasma Physics Center (RAPP Center), Ruhr-Universität Bochum, 44780 Bochum, Germany

${ }^{c}$ Max Planck Institute for Radioastronomy, 53121 Bonn, Germany

${ }^{d}$ Department of Physics \& Astronomy, University of Alabama, Tuscaloosa, AL 35487, USA

${ }^{e}$ Department of Physics, University of Florida, PO Box 118440, Gainesville, FL 32611-8440, USA

${ }^{f}$ Technische Universität Dortmund, Experimentelle Physik 5, Otto-Hahn-Strasse 4a, 44227 Dortmund, Germany

E-mail: ilja.jaroschewski@ruhr-uni-bochum.de

The first high-probability association of an extragalactic neutrino to the blazar TXS 0506+056 in 2017 identified such active galaxies as potential high-energy neutrino emitters, as expected for a long time. A detailed follow-up investigation revealed a second neutrino flare from the same blazar nearly 3 years prior, indicating a possible periodicity. A supermassive binary black hole system close to its merger explains such a periodic neutrino emission as a result of jet precession and jet interactions with surrounding molecular clouds.

We present an analytical model for predicting the arrival times of neutrino flares and gravitational waves for such systems and apply it to the blazar TXS 0506+056 under the assumption of quasiperiodic neutrino emission. We conclude that the blazar can indeed be an ongoing binary merger with the next neutrino emission possibly occurring before the end of 2021 and deliver binary properties for a successful detection of its gravitational waves by LISA.

As supermassive binary black hole mergers occur more frequently due to merging of their host galaxies, we further investigate a connection between their radiated gravitational wave energy and the diffuse astrophysical neutrino flux that is measured by IceCube. We estimate the contributions of these mergers and additionally of binary stellar mass black hole mergers in starburst galaxies to the diffuse neutrino flux.

$37^{\text {th }}$ International Cosmic Ray Conference (ICRC 2021)

July 12th-23rd, 2021

Online - Berlin, Germany

\footnotetext{
*Presenter
} 


\section{Introduction}

The first detection of gravitational wave signal from a stellar mass binary black hole (BBH) merger in 2015 and the high-probability association of a high-energy neutrino coincident with a gamma-ray flare from the direction of the blazar TXS0506+056 in 2017 illustrate the advances made in multi-messenger astrophysics in this decade alone. By now, at least three blazars are identified as possible high-energy neutrino accelerators, making active galactic nuclei (AGN) promising candidates for sources of cosmic rays (CRs) [1]. The discovery of a TeV neutrino from the direction of TXS0506+056 nearly 3 years prior to the high-energy association with no coincident gamma-ray flare indicated a periodic and complex neutrino production mechanism.

A promising explanation for such a periodic neutrino signal is a currently ongoing supermassive binary black hole (SMBBH) merger at the core of the blazar [2]. Such a merger enters its inspiral stage at sub-pc separation, after the initial merger of the host galaxies and the decrease of the separation of the two supermassive black holes (SMBHs) at their center, due to dynamical friction and interactions with molecular clouds, happened. In this inspiral stage, the binary loses most of its energy in form of radiated gravitational waves (GWs), while the previously unaligned spins of the SMBHs realign themselves leading to a change of the jet orientations, the so-called 'spin-flip' of the jets [3], which can be seen in radio data of many radio galaxies with an 'old' and a 'new', post-merger, jet orientation. During this reorientation of the jets, they precess and collide with the surrounding matter, which leads to proton-proton interactions and, as a consequence, neutrino productions [4]. In general, the jets are aligned by the end of the inspiral stage at the innermost stable orbit of the binary, after which it merges.

In the following, we derive an analytical model for prediction a neutrino signal from a precessing jet during an ongoing SMBBH merger with a spin-flip of the jet. In addition we can then predict the arrival time of the GW signal from this binary merger, based on the periodically recurring neutrinos.

\section{Jet precession Model}

\subsection{Mathematical Description}

The essential idea behind our model is shown in Fig. 1: During the spin-flip of the jets in the inspiral stage, the jets change their direction and can point at Earth, if it lies in their opening angle $\delta$. Focusing only on the stronger jet with the spin $\mathbf{S}_{\mathbf{1}}$, a first neutrino (and gamma-ray signal) can be detected at a time $t_{1}$, as the emission cone crosses the direction of Earth for the first time in the reorientation of the jets. At the time $t_{2}$, Earth would lie inside the opening angle of the jet, indicated by the blue ring-structure. However, due to the precession around the axis of the total angular momentum $\mathbf{J}$, no signal is detectable, as the jet points in another direction (orange cone). Another signal is detectable at a time $t_{3}$, during which the orange emission area of the jet points at Earth. With advancing jet reorientation, the periodicity between reoccurring signals becomes shorter until a time $t_{4}$ is reached, at which the jet no longer points towards Earth. The jet then proceeds to change its direction, until the spin $\mathbf{S}_{\mathbf{1}}$ aligns with the total angular momentum $\mathbf{J}$. The sum of the angle $\vartheta$ between $\mathbf{S}_{\mathbf{1}}$ and $\mathbf{J}$ and the angle $\alpha$ between the angular momentum $\mathbf{L}$ and $\mathbf{J}$ is constant during the spin-flip: $\vartheta+\alpha=$ const. 

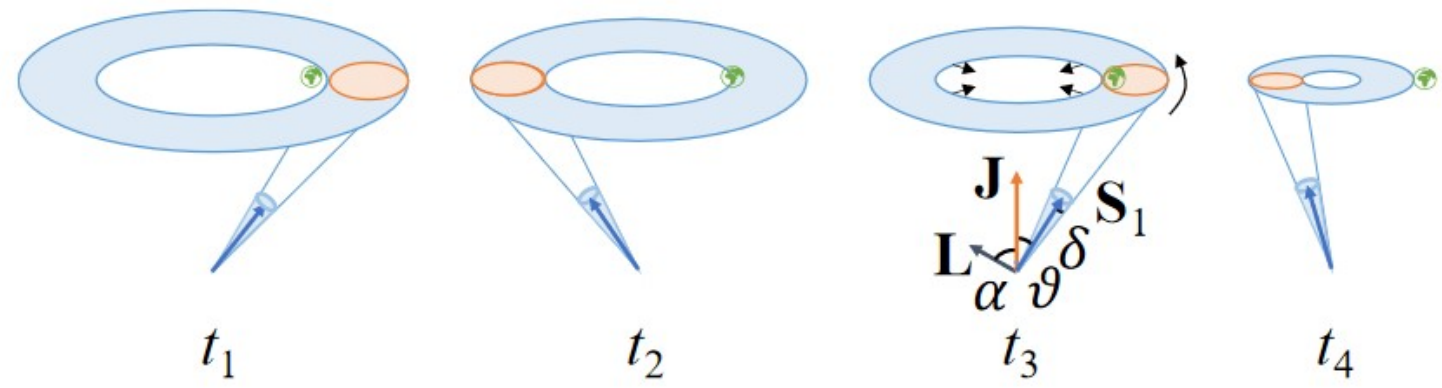

$t_{2}$

$t_{3}$

$t_{4}$

Figure 1: Schematic progress of the signal structure of a blazar (AGN) jet that recurrently points at Earth. A signal is only detected, if the orange are crosses the Earth (time $t_{3}$ ) [5].

Detection two separate neutrino emissions at different times from the same source allows a prediction on when the next emission might occur and how far the spin-flip of the jet progressed, under the assumption that the opening angle of the jet $\delta$ is known. For that, we apply the timescale of the inspiral stage, which is simultaneously the timescale for gravitational radiation and neutrino emission [3]:

$$
\Delta T_{\mathrm{GW}} \approx \frac{5 G M_{1,2}}{32 c^{3}} \cdot \frac{(1+q)^{2}}{q} \cdot \varepsilon^{-4}
$$

Here, the total mass of the SMBBH is $M_{1,2}$, the mass ratio $q=\frac{m_{2}}{m_{1}} \leq 1$ with the two masses $m_{1}$ and $m_{2}$ and $m_{1}>m_{2}$, the gravitational constant $G$ and the post-Newtonian parameter $\varepsilon \approx v / c$. This time scale can also be denoted as the remaining time until the merger with $\Delta T_{\mathrm{GW}}+t=t_{\text {merger }}$ and $t$ being the progressed time in the past, during which a part of the jet spin-flip occurred. This approach is sufficient until a post-Newtonian parameter $\varepsilon \lesssim 0.1$, which corresponds to $\Delta T_{\mathrm{GW}} \gtrsim 0.5-2.4$ years before the merger, so sufficient for our model. The angle $\phi$ of the precession of the spin $\mathbf{S}_{\mathbf{1}}$ around $\mathbf{J}$ can then be expressed as [5]:

$$
\phi\left(\Delta T_{\mathrm{GW}}\right)=-8\left(\frac{5 c}{32 G^{1 / 3} M_{1,2}{ }^{1 / 3}} \cdot \frac{(1+q)^{2}}{q}\right)^{3 / 4} \Delta T_{\mathrm{GW}}^{1 / 4}+\phi_{0},
$$

with the integration constant $\phi_{0}$. Using this relation, we can determine the remaining time until merger $\Delta T_{\mathrm{GW}}$, which delivers the same angle $\phi$ after a periodic precession of the jet $t_{\mathrm{Jet}}$ :

$$
\phi\left(\Delta T_{\mathrm{GW}}\right)=\phi\left(\Delta T_{\mathrm{GW}}+t_{\mathrm{Jet}}\right)=\phi_{\mathrm{Earth}} \pm \delta .
$$

\subsection{Prediction for TX0506+056}

Applying this model on the blazar TXS0506+056 results in Fig. 2. As this blazar is the only one, which caused two neutrino detections, both in 2014/2015 [6] and September 2017 [7], the periodicity was determined as $t_{\mathrm{Jet}}=2.78 \pm 0.15$ years. Other parameters that entered this model are the mass of the central region of $3 \cdot 10^{8} M_{\odot}$, a mass ratio of the binary between $q=1 / 3$ and $q=1 / 30$ [3], as well as an opening angle of the jet of $\delta=5^{\circ}$.

Fig. 2 shows the next two predicted episodes of neutrino emission from this blazar in dependence of its mass ratio $q$. The blue area marks the time, during which the jet will show in the direction 


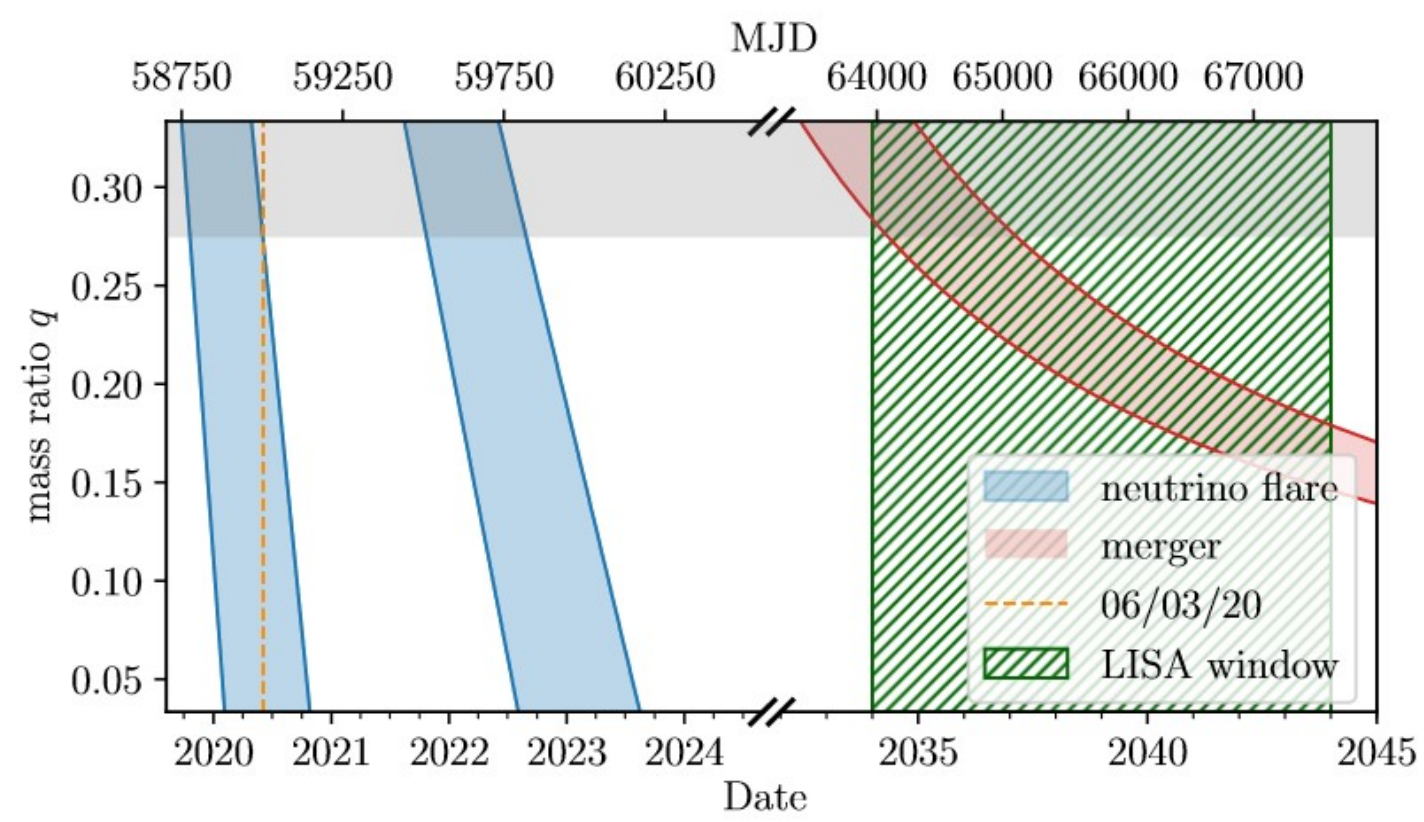

Figure 2: Predictions for neutrino and gravitational wave detections from TXS0506+056 in dependence of the mass ratio $q$. The next two periods of neutrino emissions are illustrated in blue, while the arrival times of GWs are shown in red. The dashed orange line marks June 3rd 2020 [5].

of Earth, considering the uncertainty in the periodicity of the previous signals. The moment of the SMBBH merger and thus the arrival time of its GWs during the merger are displayed in red. All parameter combinations predict a merger in the next $15-120$ years. For a mass ratio $q \geq 0.15$, the gravitational wave detector LISA will be able to detect the GWs during its observational run.

\section{Connection between Neutrinos and Gravitational Waves}

In order to establish a connection between the radiated GW energy during a SMBBH merger and the emitted energy in form of neutrinos, we assume that the jets encounter molecular and dusty clouds at all time during their spin-flip in the inspiral stage. Only then is the time scale for neutrino production the same as the time scale for GW radiation and we can make the connection

$$
E_{v}^{\mathrm{total}}=f_{\mathrm{SMBBH}}^{v} \cdot E_{\mathrm{GW}},
$$

with the energy fraction $f_{\text {SMBBH }}^{v}$ that neutrinos receive with their total energy $E_{v}^{\text {total }}$ compared to the total radiated GW energy $E_{\mathrm{GW}}$. The diffuse neutrino flux at Earth can then be expressed with the total radiated gravitational wave energy as

$$
E_{v}^{p} \Phi\left(E_{v}\right)=\underbrace{\kappa_{p} \cdot f_{\mathrm{SMBBH}}^{v} \cdot c \cdot t_{\mathrm{H}}}_{\zeta_{c}} \cdot \xi_{z} \cdot \underbrace{\int_{M} \frac{\mathrm{d} E_{\mathrm{GW}}}{\mathrm{d} t} \cdot g(M) \mathrm{d} M}_{\zeta_{M}},
$$

when weighted with an energy with the spectral slope $p$. This relation was derived using the approach by [8] and converting the luminosity dependency into a mass dependency. The factor $\kappa_{p}$ 
is an integration constant and takes the values

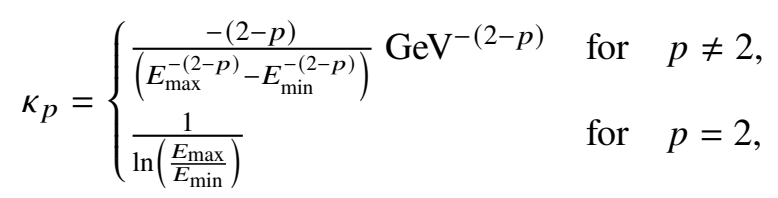

with the minimal and maximal energies of neutrinos $E_{\min }$ and $E_{\max }$, respectively. We applied the diffuse astrophysical neutrino flux with ten years of IceCube data by [9] with a spectral slope of $p=2.28_{-0.09}^{+0.08}$ and set the neutrino energy range to $E_{\min }=100 \mathrm{GeV}$ and $E_{\max }=100 \mathrm{PeV}$, to account for the part of the spectrum, which is not detected by IceCube.

The activity integral $\xi_{z}$ contains the source evolution density of SMBBHs and is dependent on cosmological constants. We applied a $\Lambda$-CDM Universe with the current Planck data [10] and estimated the activity integral to a value of $\xi_{z}=2.4_{-0.5}^{+8.3}$, as the evolution of SMBBH mergers differs from that of AGN in general.

In order to determine the mass dependent integral $\zeta_{M}$, we describe $g(M)$ as a Schechter function $n(M)$ of the SMBH mass distribution divided by the mass $M$ with

$$
n(M)=\rho_{0} \cdot\left(\frac{M}{M_{\star}}\right)^{-\alpha} \cdot \exp \left[-\left(\frac{M}{M_{\star}}\right)^{\beta}\right] .
$$

Comparing the coefficients with the gravitational focusing cross section by [11] in the differential representation, which results in the values $\alpha=1$ and $\beta=1 / 3$, we plot the Schechter function to the integrated SMBH mass function by [12] and receive the parameters $\rho_{0}=10^{-4} \mathrm{Mpc}^{-3}$ and $M_{\star}=6.9 \cdot 10^{8} \mathrm{M}_{\odot}$. This SMBH mass distribution contains $2.4 \cdot 10^{4 \pm 0.4} \mathrm{SMBHs}$ in a radius of $100 \mathrm{Mpc}$, resulting in an extrapolated density of $n_{\mathrm{SMBH}}=5.73 \cdot 10^{6 \pm 0.4} \mathrm{Gpc}^{-3}$ (see [12] for more details). Approximating the radiated GW energy during each SMBBH merger with $10 \%$ of the binaries chirp mass $\mathcal{M}$, the total GW energy during the formation of each SMBH is expressed as

$$
E_{\mathrm{GW}}=\frac{k(q)}{(1-k(q))} \cdot M \cdot c^{2},=h(q) \cdot M \cdot c^{2}
$$

with the mass ratio of the binary $q=m_{1} / m_{2} \leq 1$, their combined mass $M_{1,2}=m_{1}+m_{2}$, the merged SMBH mass $M$ and the factor $k(q)=0.1 \cdot \sqrt[5]{\frac{q^{3}}{(1+q)^{6}}}$, so that $0.1 \cdot \mathcal{M}=k(q) \cdot M_{1,2}$. Now, a relation between the fraction $f_{\mathrm{SMBBH}}^{\nu}$ of GW energy that neutrinos receive and the rate of SMBBH mergers is established by expressing $\zeta_{M}$ in dependency of the rate $R_{\mathrm{SMBH}}$ :

$$
\zeta_{M} \cdot \frac{1}{h(q)}=\int_{M} \frac{d\left(M \cdot c^{2}\right)}{d t} \cdot g(M) d M=\langle M\rangle \cdot c^{2} \cdot \frac{n_{\mathrm{SMBH}}}{\bar{t}_{\text {total }}}=\langle M\rangle \cdot c^{2} \cdot R_{\mathrm{SMBH}},
$$

with the mean mass in the distribution $\langle M\rangle=4.9 \cdot 10^{7} \mathrm{M}_{\odot}$ and the mean time for one SMBBH merger $\bar{t}_{\text {total }}$. In doing so, different SMBBH merger rates can be set in relation to $f_{\mathrm{SMBBH}}^{v}$ and investigated. This was done in Fig. 3. Here, two mass ratios are investigated, $q=1 / 3$ and $q=1 / 30$, as most mergers happen inside this mass ratio range [3]. The diamond shaped points are constructed using the former mass ratio, while stars apply the later. All values for the rate and the energy fraction $f_{\text {SMBBH }}^{v}$ are presented in Table 1. The inspiral time model by [3] is presented in violet and represents the energy fraction that neutrinos receive compared to GWs. However, its rate $R_{\text {ins }}$, captures only 


\begin{tabular}{|c|c|c|c|c|}
\hline \multirow[t]{2}{*}{ model } & \multicolumn{2}{|c|}{$\begin{array}{l}R_{\text {insp }} / 10^{ \pm 0.4} \text { in } \\
\mathrm{Gpc}^{-3} \mathrm{yr}^{-1}\end{array}$} & \multicolumn{2}{|c|}{$f_{\mathrm{SMBBH}}^{v} / 10^{-6}$} \\
\hline & $q=1 / 3$ & $q=1 / 30$ & $q=1 / 3$ & $q=1 / 30$ \\
\hline \multirow[t]{2}{*}{ Gergely and Biermann (2009) } & 2.85 & 0.47 & $1.50_{-0.86}^{+6.49} \cdot 10^{-3}$ & $2.71_{-1.08}^{+11.70} \cdot 10^{-2}$ \\
\hline & \multicolumn{2}{|c|}{$\begin{array}{l}R_{\mathrm{SMBH}} / 10^{-3 \pm 0.4} \text { in } \\
\mathrm{Gpc}^{-3} \mathrm{yr}^{-1}\end{array}$} & \multicolumn{2}{|c|}{$f_{\mathrm{SMBBH}, \mathrm{gal}}^{v} / 10^{-6}$} \\
\hline $\begin{array}{l}\langle M\rangle=4.90 \cdot 10^{7} M_{\odot} \quad(6[q= \\
1 / 3], 8[q=1 / 30] \text { mergers })\end{array}$ & 2.32 & 3.86 & $1.84_{-1.06}^{+7.98}$ & $3.32_{-1.91}^{+14.39}$ \\
\hline \multirow{2}{*}{$\begin{array}{l}M_{\text {median }}=1.45 \cdot 10^{8} M_{\odot}(10[q= \\
1 / 3], 13[q=1 / 30] \text { mergers })\end{array}$} & 3.09 & 5.02 & $1.38_{-0.79}^{+5.98}$ & $2.56_{-1.47}^{+11.07}$ \\
\hline & \multicolumn{2}{|c|}{$R_{\mathrm{BBH}}$ in $\mathrm{Gpc}^{-3} \mathrm{yr}^{-1}$} & \multicolumn{2}{|c|}{$f_{\mathrm{BBH}}^{v} / 10^{-6}$} \\
\hline $\begin{array}{l}\text { LIGO/Virgo BBH merger rate } \\
\text { ( } 90 \% \text { credible interval), Abbott } \\
\text { et al. }(2020 \mathrm{~b})\end{array}$ & \multicolumn{2}{|c|}{$23.9_{-8.6}^{+14.3}$} & \multicolumn{2}{|c|}{$1.29_{-0.98}^{+1.40} \cdot 10^{1}$} \\
\hline $\begin{array}{l}\text { Rate of events similar to } \\
41.9+58, \text { Biermann et al. }(2018)\end{array}$ & \multicolumn{2}{|c|}{$40_{-20}^{+40}$} & \multicolumn{2}{|c|}{$7.74_{-6.44}^{+10.31}$} \\
\hline
\end{tabular}

Table 1: Values for the neutrino production rate in SMBBHs $R_{i n s p}$, the detection rate of the GWs from SMBBHs $R_{\mathrm{SMBH}}$ and the stellar mass $\mathrm{BBH}$ detection rate $R_{\mathrm{BBH}}$ with the respective neutrino energy fraction from the radiated gravitational wave energy.

SMBBH mergers, once they entered the inspiral stage, the previous galaxy merger and other stages in the binary evolution are neglected.

In contrast, the rates $R_{\mathrm{SMBH}}$, indicated as red and green points, capture all timescales of a SMBBH merger, including the merger of the host galaxies. For that, a simple iteration was performed, which delivered the number of necessary mergers $i$ in order to receive the mean mass $\langle M\rangle$ and median mass $M_{\text {median }}=9.03 \cdot 10^{8} \mathrm{M}_{\odot}$ of the $\mathrm{SMBH}$ mass distribution, beginning with the mass $m_{1}=3 \cdot 10^{6} \mathrm{M}_{\odot}$ :

$$
M=\left[\left(1+\eta_{\mathrm{acc}}-k(q)\right) \cdot(1+q)\right]^{i} \cdot m_{1}
$$

The factor $\eta_{\text {acc }}$ captures a constant accretion percentage between each SMBBH merger and was set to $\eta_{\text {acc }}=1 / 3$, so that the mass gain via accretion is the same as the mass gain via the highest mass ratio $q=1 / 3$. For the mean mass $\langle M\rangle, 6$ mergers with a mass ratio of $q=1 / 3$ and 8 mergers for the mass ratio $1 / 30$ are required, while the median mass required 10 and 13 mergers, respectively. These mergers happened in the redshift range $z \in[1,3]$ in galaxy groups and clusters, as the galaxy density was the highest during this cosmic period. The factor $f_{\text {SMBBH,gal }}^{v}$ captures the effective fraction of GW energy that neutrinos receive, as it contains timescales, during which no neutrinos or gravitational waves are produced. However, the factor $R_{\mathrm{SMBH}}$ is the detection rate of GWs from SMBBH mergers. 


\begin{tabular}{|c|c|c|c|c|c|}
\hline & $\begin{array}{l}\text { SMBBH mergers } \\
\text { with } q=1 / 3\end{array}$ & $-\cdots$ & $\begin{array}{l}\text { SMBBH mergers } \\
\text { with } q=1 / 30\end{array}$ & & $\begin{array}{l}\text { BBH mergers with } \\
<E_{G W}>=2.85 M_{\odot}\end{array}$ \\
\hline 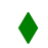 & $\bar{t}_{\text {total }}(\langle M\rangle, 1 / 3)=2.47 \mathrm{Gyr}$ & $\star$ & $\bar{t}_{\text {total }}(\langle M\rangle, 1 / 30)=1.48 \mathrm{Gyr}$ & $\bullet$ & Biermann et al. (2018) \\
\hline 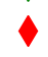 & $\bar{t}_{\text {total }}\left(M_{\text {median }}, 1 / 3\right)=1.86 \mathrm{Gyr}$ & $\star$ & $\bar{t}_{\text {total }}\left(M_{\text {median }}, 1 / 30\right)=1.14 \mathrm{Gyr}$ & $\bullet$ & $\begin{array}{l}\text { LIGO/Virgo BBH merger rate, } \\
\text { LIGO and Virgo et al. (2021) }\end{array}$ \\
\hline $\boldsymbol{\nabla}$ & $\begin{array}{l}\text { Gergely and Biermann (2009), } \\
q=1 / 3\end{array}$ & $\star$ & $\begin{array}{l}\text { Gergely and Biermann (2009), } \\
q=1 / 30\end{array}$ & - & de Vries et al. (2017) \\
\hline
\end{tabular}

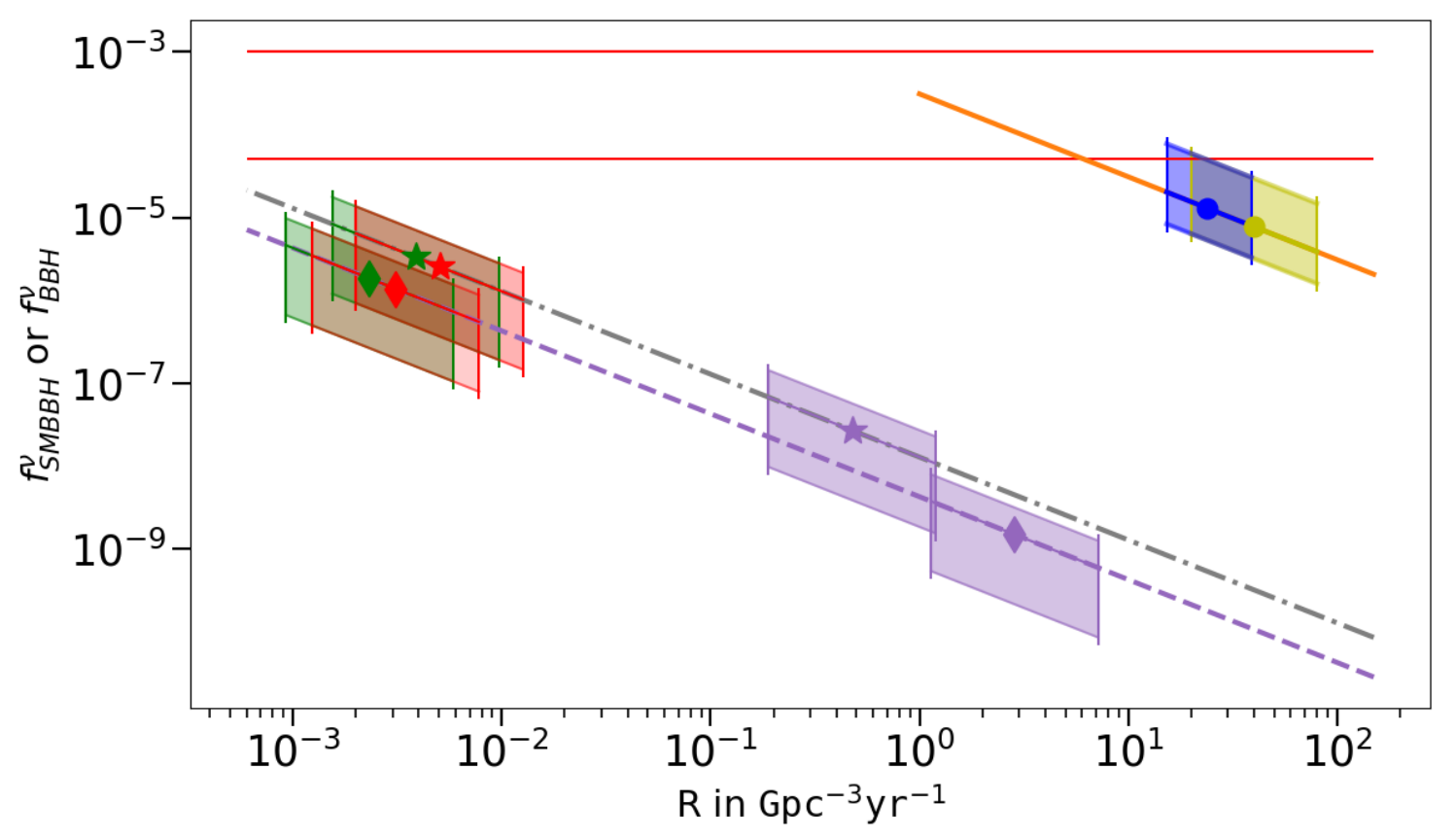

Figure 3: Fraction of gravitational wave energy that goes into neutrinos during SMBBH merger $f_{\mathrm{SMBBH}}^{\nu}$ and stellar mass BBH merger $f_{\mathrm{BBH}}^{v}$ events with the associated rates. Stellar mass BBH merger rates are marked as dots, while SMBBH and inspiral rates are marked with diamonds for a mass ratio of $q=1 / 3$ and stars for $q=1 / 30$. Red horizontal lines mark comparative values for $f_{\mathrm{BBH}}^{v}[13]$.

In addition, Fig. 3 contains the stellar mass binary black hole (BBH) merger rates, since these black holes can possess a strong relativistic jet and merge partially with a spin-flip as well [14], as the data from the Gravitational-Wave Transient Catalogue GWTC-2 shows [15]. We compared two reference merger rates of BBHs: the inferred merging BBH detection rate by the LIGO and Virgo Collaborations with $90 \%$ confidence level [16] and an estimation for this rate by radio observations by [14]. Since the Pierre Auger Collaboration anisotropy study of ultra-high energy cosmic rays hints that $10 \%$ originate from starburst galaxies and the rest from AGN [17], we weighted the neutrino fraction from GW energy in stellar mass BBH mergers $f_{\mathrm{BBH}}^{v}$ with 0.1 and the fraction in SMBBH mergers with 0.9. As a result, the same fraction of the emitted GW energy goes into neutrinos during SMBBH mergers and stellar mass BBH mergers.

\section{Summary and Outlook}

We developed an analytical model for predicting the neutrino emission and gravitational wave radiation of a supermassive binary black hole merger and applied it on the blazar TXS0506+056. We predict the next two episodes of neutrino flares, with the first already happened before the 
end of 2020, which could be revealed by a blind IceCube data analysis in the future, if the signal was strong enough. A study on possible neutrino emission from the SMBBH merger population revealed that neutrinos receive approximately the same ratio of GW energy in SMBBH mergers $\left(f_{\mathrm{SMBBH}}^{v} \approx(0.59-17.71) \cdot 10^{-6}\right)$ as in stellar mass BBH mergers $\left(f_{\mathrm{BBH}}^{v} \approx(1.23-26.90) \cdot 10^{-6}\right)$, if the SMBBH mergers make up $90 \%$ and stellar mass BBH mergers $10 \%$ of the diffusive astrophysical neutrino flux that is measured by IceCube. This could imply that this diffusive neutrino flux is made up by a combination of the two source classes.

Both models will be improved with future detections of gravitational waves from stellar mass binary black hole mergers by the LIGO and Virgo Collaborations. A non-disappearing eccentricity component of the $\mathrm{BH}$ orbit during the spin-flip will also extend the analytical neutrino prediction model.

\section{References}

[1] Kun, E., Bartos, I., Becker Tjus, J. et al., 2021, In: ApJL (911) L18, [arXiv/2009.09792]

[2] Britzen, S., Fendt, C., Böttcher, M. et al., 2019, In: A\&A (630) A103

[3] Gergely, L. Á. and Biermann, P. L., 2009, In: ApJ (697) 1621, [arXiv/0704.1968]

[4] Becker Tjus, J. and Merten, L., 2020, In: Physics Reports (872) 1, [arXiv/2002.00964]

[5] de Bruijn, O., Bartos, I., Biermann, P. L. and Becker Tjus, J., 2020, In: ApJL (905) L13, [arXiv/2006.11288]

[6] IceCube Collaboration et al., 2018, In: Science (361) 147, [arXiv/1807.08794]

[7] IceCube Collaboration et al., 2018, In: Science (361) 1378, [arXiv/1807.08816]

[8] Becker, J. K., 2008, In: Physics Reports (458) 173, [arXiv/0710.1557]

[9] Stettner, J., 2019, In: ICRC 2019, [arXiv/1908.09551]

[10] Planck Collaboration et al., 2020, In: A\&A (641) A6, [arXiv/1807.06209]

[11] Silk, J. and Takahashi, T., 1979, In: ApJ (229) 242

[12] Caramete, L. I. and Biermann, P. L., 2010, In: A\&A (521) A55, [arXiv/0908.2764]

[13] de Vries, K. D., de Wasseige, G., Frère, J.-M., Vereecken, M., 2017, In: Phys. Rev. D (96) 3003, [arXiv/1709.04880]

[14] Biermann, P. L. et al., 2018, In: Advances in Space Research (62) 2773, [arXiv/1803.10752]

[15] The LIGO Scientific and Virgo Collaboration et al., 2021, In: Phys. Rev. X (11) 1053, [arXiv/2010.14527]

[16] The LIGO Scientific and Virgo Collaboration et al., 2021, In: ApJL (913) L7, [arXiv/2010.14533]

[17] Pierre Auger Collaboration et al., 2018, In: ApJL (853) L29, [arXiv/1801.06160] 